\title{
General Survey of Glucose and Lipid Metabolism in Spontaneously Hypertensive Rats (SHR)
}

\author{
Kiro Shimamoto, M.D., Akio Teraoka, Ph.D., Hisashi Iwatsuka, Ph.D., \\ and Ziro Suzuoki, Ph.D.
}

Analysis of serum components and some clinical tests were performed in 3 substrains of $\operatorname{SHR}\left(\mathrm{A}_{1}-\mathrm{sb}, \mathrm{B}\right.$, and $\mathrm{C}$ ) and Wistar/Kyoto, as controls, to investigate metabolic abnormalities in SHR.

There was no difference in the serum glucose and free fatty acid (FFA) levels between SHR substrains and controls. The serum cholesterol level was decreased in SHR substrains. These 3 metabolic parameters did not differ among 3 substrains of SHR.

The serum triglyceride level was increased in $\mathrm{A}_{1}{ }^{-\mathrm{sb}}$, but not in $\mathrm{B}$ and $\mathrm{C}$, in comparison with controls. This difference was disappeared after overnight fasting. When olive oil was administered orally, $A_{1}{ }^{-s b}$ showed more remarkable increase in the triglyceride level than others. Therefore, the elevated triglyceride level of this substrain seems to be due to an increase of exogenous lipoprotein.

The serum insulin level was significantly lower in SHR substrains than controls in either fed or fasted state. Oral glucose tolerance test was performed on fasted rats. All of SHR substrains showed intolerance to glucose accompanying low response of insulin release, when compared with controls. Response of FFA to glucose loading was also impaired in SHR. These findings suggest that SHR have functionally impaired pancreatic $\beta$ cells which results in disturbance of glucose and FFA metabolism.

These studies indicated that SHR could be distinguished from Wistar/ Kyoto, by some features, not only in the blood pressure, but also in metabolism. The metabolic differences, indicated here, seem to depend upon genetic variation which might occur during the selection of animals to establish SHR. Such genetic variation may exist among SHR substrains, as suggested by difference in the triglyceride level between $A_{1}{ }^{-s b}$ and others.

From the Biological Research Laboratories, Central Research Division, Takeda Chemical Industries, Ltd., Osaka. 